\title{
Cardiac catheterization
}

\author{
Graham Miller ${ }^{1}$
}

Since 194I when Cournand and Ranges developed Forssmann's (1929) original observation, cardiac catheterization has become a technique essential to the practice of cardiology. Now, 30 years later, it may be appropriate to review the practice of catheterization today; who should perform the procedure and on what patients? What techniques are of value and where should catheterization laboratories be built? Traditionally the procedure has been performed by cardiologists and this is proper, since adequate performance of cardiac catheterization requires a wide knowledge of cardiology and, in turn, clinical practice is benefited by an understanding of the results which can be obtained in the catheterization laboratory. Occasionally some of the techniques of cardiac catheterization have been practised by radiologists who had the skills required for approaches to the left heart when this was a new procedure; unfortunately this has, on occasion, led to an arbitrary division of the procedure based on technical skills and not on the haemodynamic and physiological problems which should be approached as a whole. Right and left heart catheterizations should not be separate procedures, and angiocardiography is an integral, not separate, part of the investigation. Indeed angiocardiography can be performed intelligently only after a full understanding of the physiological abnormality has been obtained from the haemodynamic findings. This is not to say that radiologists should not perform cardiac catheterizations - on the contrary the procedure may be performed by physicians trained in many disciplines, provided they have the necessary understanding of cardiology and cardiac physiology - they can then learn the required technical skills.

The contribution of radiology to cardiac catheterization has been very great, chiefly, perhaps, in providing descriptions of abnormal anatomy. But radiological techniques can

${ }^{1}$ Address: Brompton Hospital, London S.W.3. also provide information of a physiological nature; a striking example is the use of angiographic methods to provide measurements of left ventricular volume and, from this, of contractility, wall thickness and stress, and so on. Such investigations have, to a large extent, been the work of radiologists and are a prototype of the way in which radiology can contribute to cardiology, over and above simple anatomical descriptions.

A division which is becoming increasingly common is that between paediatric and adult cardiology; cardiac catheterization in neonates and infants with complex congenital malformations requires special technical skills, extensive knowledge of the wide variety of conditions to be met with, and, importantly, the ability to think fast on one's feet. Not everyone who can catheterize adult patients has these skills and the procedure should only be performed by those who have - none the less, a complete separation between paediatric and adult cardiology would be unfortunate; both groups of cardiologists can benefit from understanding the problems of the other, and the catheterization laboratory, looking at all age groups and abnormalities from a physiological viewpoint, can become a meeting point for the two groups, where the discipline of haemodynamics provides a unifying force and an excellent training for both junior and senior cardiologists.

Perhaps the most widely accepted and yet the least rational division is that between cardiac and respiratory physiology. To the physiologist the heart and lungs are one organ while the clinician recognizes how many conditions there are in which cardiac and pulmonary disease interreact. Yet it is unusual to find facilities for the investigation of heart and lung function together in one laboratory or in one department. Quite apart from the rationalization in equipment and staff that would result from such an approach, both groups of physicians would benefit enormously from contact with each other and from the 
ability to study both heart and lungs simultaneously using all the facilities, including cardiac catheterization, presently available in separate departments.

The indications for catheterization are constantly changing. To a large extent these changes are dictated by advances in cardiac surgery; thus the increasing number of patients submitted to coronary arteriography and of infants studied for congenital cardiac anomalies reflects the advances that have been made in the surgery of ischaemic and of congenital heart disease. Advances in surgery have also resulted in changing techniques of catheterization. Thus, when mitral valvotomy was the only effective operation for rheumatic heart disease considerable effort was expended on techniques which attempted to differentiate between dominant stenosis and dominant incompetence of the mitral valve. Today, when there is an increasing tendency for mitral valvotomy to be performed on cardiopulmonary bypass and when valve replacement is readily available, the emphasis has changed from assessing incompetence/stenosis to assessing how many valves are diseased and need replacement. As a result left ventriculography may now be performed less for the purpose of assessing the severity of mitral incompetence as for assessing myocardial function. The assessment of myocardial function is a relatively new but increasingly important part of the work of a catheterization laboratory, as it is increasingly recognized as an important determinant of prognosis. The need to assess myocardial function has led to the development of new techniques such as intracardiac manometry, aortic blood velocity measurement, and so on.

Cardiac surgery creates its own problems which have to be studied in the catheterization laboratory; thrombosis and incompetence or detachment of prosthetic valves are examples. Patients with these complications may be acutely ill, and the study may present technical problems - as when entry to the left ventricle is guarded by mitral and aortic ball valve prostheses. Complications of surgery such as these may thus demand new techniques of study and may present with unfamiliar haemodynamic disturbances which we must learn to recognize-just as we have to learn a new set of physical signs relating to these complications.

A cardiac laboratory today finds that it is studying an increasing number of acutely ill patients, yet among the traditional approaches to cardiac catheterization is the view that it is a 'cold' procedure for which the patient must be prepared by medical treatment and which is contraindicated in the very sick. Any catheterization laboratory which is concerned with the study of neonates will soon realize that such an approach is quite impossible; as Lees (1969) has pointed out neonates in whom heart failure occurs in the first 2 or 3 days of life usually follow a relentlessly downhill course and 'if the lesion is to be surgically corrected, diagnostic studies should be performed within a few hours'. Such neonates with severe congenital heart disease undoubtedly form a high risk group and there may be a temptation to perform only a minimal study or even to perform angiocardiography alone in an attempt to reduce the risk. Such a policy is misguided; the correct management of such patients almost always requires full knowledge of the defects present and an incomplete study either leads to incorrect management or to the need for a further study with further risks. Lambert and co-workers (Lambert, Canent, and Hohn, 1966) have emphasized that in this group of patients full haemodynamic and angiographic studies are mandatory despite the high risk and low salvage rate to be expected. In a co-operative study of the risks of catheterization based on more than 12,000 procedures the complication and mortality rates in patients under 30 days of age were 15.3 and 6.2 per cent respectively - in contrast to the overall complication and mortality rates of 3.6 and 0.44 per cent (Braunwald and Gorlin, 1968; Braunwald, 1968). A leading cause of death in the infant group was perforation of the heart or great vessels - emphasizing how important it is to reduce catheter manipulation to a minimum in these patients. Reduction of these risks requires sophisticated instrumentation and a skilled and disciplined catheterization team able to obtain and interpret information rapidly while paying attention to acid-base balance, heat loss, and other factors which may adversely affect the infant during the course of the study. The instrumentation should include some system of 'on-line' analysis of oxygen saturation to avoid removing (and replacing) blood and to provide an immediate reading of oxygen saturation before the catheter is moved from the sampling site. With this facility it should seldom be necessary to readvance a catheter to a previously entered site in order to obtain information that has been missed or whose importance has not been recognized. Only by having a continuous feedback of information can the operator proceed logically and rapidly with the investigation. With proper instrumentation and in experienced hands there are few cases of heart disease in neonates in whom a full study cannot be completed in, at most, I to $I \frac{1}{2}$ hours. 
The risks of catheterization in the neonatal period may have been exaggerated; it is, perhaps, unfair to attribute to previous catheterization a death occurring in an infant with a condition such as aortic atresia which is, anyway, associated with a 75 per cent mortality in the first week of life (Lambert et al., 1966).

Neonates and infants with severe congenital heart disease are, of course, not the only group of critically ill patients in whom the risks of catheterization may appear prohibitive adults with the complications of valve surgery and patients with recent myocardial infarction , or massive pulmonary embolism are other examples. In such patients, too, investigation is mandatory if treatment depends on the findings at catheterization and can be shown to reduce mortality. With advances in surgical technique it is probable that more and more such high risk patients will be studied by cardiac catheterization. Clearly a laboratory undertaking the study of such patients must be prepared to operate on a 24 hour per day, 7 day per week basis. It requires a surgical 'outlet' and is likely to be situated in a centre where open heart surgery is undertaken as a more than occasional part of the work of the hospital. Such a laboratory is enormously expensive to equip and to run, and requires a large staff of highly trained technicians - a group in which there is a serious recruiting problem, as might be expected when the levels of pay are so abysmally low. When a laboratory is working in association with a cardiac surgical team, it is likely that the techniques employed will be geared to provide those bits of information which are required by a particular group of surgeons. Under these circumstances a previous study performed elsewhere, though apparently satisfactory, may have to be repeated before the surgical team can be satisfied that they have all the necessary information. Such a situation may be distressing to the patient, or his parents, and a second study may even be more difficult technically if, for example, veins have been sacrificed at the first study. All of these considerations suggest that catheterization laboratories should not be allowed to proliferate and should, perhaps, be limited to those centres serving a population of $I$ to 3 million where open heart surgery can be economically practised and where a laboratory performing $400-$ 500 catheterizations a year makes economical use of expensive equipment (Royal College of Physicians, Royal College of Surgeons Joint Cardiology Committee Report, 1967).

At a time when more senior cardiac registrars are being trained than can be accommodated in major cardiac centres, a suggestion that catheterization laboratories should be limited in number is bound to be resisted. Cardiologists who have been trained in the technique rightly regard catheterization as an essential tool without which they cannot adequately practise their speciality. Perhaps, however, an answer to these mutually incompatible views may be provided by the recent development of new techniques of 'bedside' catheterization and the development of external aids to diagnosis. Thus the technique of micro-catheterization developed by Bradley (1964) and the Swan-Ganz flow-guided catheter (Swan et al., 1970) may provide a considerable amount of diagnostic information without the need for expensive $x$-ray equipment and without important distress to the patient. In particular the Swan-Ganz catheter, which permits wedge pressures to be obtained and through which multiple samples may be withdrawn with ease, permits considerable diagnostic information to be obtained both in the emergency situation and in the 'cold' case. External aids to diagnosis such as ultrasound and phonocardiography also enable the cardiologist, working outside a major cardiac unit, to sort out those patients who should be referred for cardiac surgery and, possibly, fuller diagnostic studies, from those who are properly treated at the 'peripheral' hospital.

Such a scheme is, it seems, appropriate to the present practice of cardiology but, and it is an important proviso, the situation could be radically altered by recent trends in the diagnosis and treatment of ischaemic heart disease. If revascularization procedures are shown to be of benefit in a large number of patients suffering from coronary artery disease and if such procedures are also shown to be of value in reducing the mortality from 'pump failure' after myocardial infarction, then few of the existing major cardiac centres could cope with the potential increased load of investigations. It is conceivable that they could do so by a large expansion in diagnostic facilities, bearing in mind that it is possible for some 600 to 700 procedures to be performed per annum in one catheterization laboratory. Alternatively, we might see a large number of centres developing the techniques of coronary arteriography and left heart catheterization as an almost entirely radiological procedure.

Finally, in discussing the role of the catheterization laboratory, it is important to consider its place in the performance of clinical research. Catheterization is a powerful tool for many research purposes, providing objective measurements of haemodynamic change after drugs or after surgical or other interventions. We must always beware, however, of suc- 
cumbing to the temptation to perform studies simply because catheterization is such a tempting tool that we cannot resist using it. While the responsibility for deciding on the ethical acceptability of a proposed study rests with the clinician, the Medical Research Council in its report on 'Responsibility in investigation on human subjects' (Medical Research Council Reports 1962-1963) sets out some useful guidelines. In particular, the distinction between 'procedures contributing to the benefit of the individual' and those 'which are not of direct benefit to the individual being studied' is an important one. In the former case the patient's consent to the procedure may be assumed, but in the latter case it is always necessary that 'the true and informed consent of the subject is explicitly obtained'. A particular difficulty arises in the case of children; in the strict view of the law 'parents and guardians of minors cannot give consent on their behalf to any procedures which are of no particular benefit to them and which may cause some risk of harm'. Thus 'where the subject is below the age of 12 years information requiring the performance of any procedure involving his body would need to be obtained incidentally without altering the nature of a procedure intended for his individual benefit'. The responsibility for ensuring that irreproachable ethical standards are maintained lies in many places. It lies, for example, with the head of a department or the director of a catheterization laboratory; it is also the view of the Medical Research Council that editors of medical journals have a responsibility in this matter and will refuse for publication those articles reporting investigations in which the ethical standards are less than irreproachable - this is also the view of the Editor of the British Heart fournal.

\section{References}

Bradley, R. D. (1964). Diagnostic right-heart catheterization with miniature catheters in severely ill patients. Lancet, 2, 94I.

Braunwald, E. (1968). Deaths related to cardiac catheterization. Circulation, 37, Suppl. 3, I7.

Braunwald, E., and Gorlin, R. (1968). Total population studied, procedures employed and incidence of complications. Circulation, 37, Suppl. 3, 8.

Cournand, A., and Ranges, H. A. (I94I). Catheterization of the right auricle in man. Proceedings of the Society for Experimental Biology and Medicine, 46, 462.

Forssmann, W. (1929). Die Sondierung des rechten Herzens. Klinische Wochenschrift, 8, 2085.

Lambert, E. C., Canent, R. V., and Hohn, A. R. (1966). Congenital cardiac anomalies in the newborn. Pediatrics, 37, 343.

Lees, M. H. (1969). Heart failure in the newborn infant. Fournal of Pediatrics, 75, 139.

Medical Research Council (1962-1963). Responsibility in Investigations on Human Subjects, p. 21.

Royal College of Physicians of London, Royal College of Surgeons of England (1967). Joint Cardiology Committee Report, October 1967. Royal College of Physicians, London.

Swan, H. J. C., Ganz, W., Forrester, J., Marcus, H., Diamond, G., and Chonette, D. (I970). Cardiac catheterization with a flow-guided balloon-tipped catheter. New England fournal of Medicine, 283, 447. 